\title{
Failure Analysis of Firearm and Ammunition Components
}

\author{
Frederick E. Schmidt, Jr., * Michael J. Danko, * and Kent L. Johnson* \\ * Engineering Systems Incorporated, 3851 Exchange Avenue, Aurora, IL 60504
}

The general topic of impact damage to metals and alloys and more specifically impact fatigue at temperatures below 300 degrees centigrade has become a major subject in the forensic arena. The gap in testing knowledge more or less spans the mechanical testing and examination of Charpy "vee-notch" ASTM samples compared to ASTM fracture mechanics tests. The microscopist examining field service failures has the difficult task of first cleaning the fracture surfaces (1) and then eliminating potential environmental contributions e.g. hydrogen or stress corrosion effects to the failure mode.

One other handicap that sometimes restricts a forensic investigator and should be avoided is the temptation to immediately go to a scanning electron microscope (SEM) without first making a determination of the composition of the alloy and microstructure. A previous study has documented the materials processing options and morphological relationship for the medium carbon, resulfurized steel that is the subject of this analysis $(2,3)$. A brief summary of these papers indicates the subject steel has been hot worked above $\sim 700$ degrees centigrade, is a $50 \%$ ferrite and 50\% pearlite at Rockwell C 20 to 28.

In this instance the incrementally hot forged (1100 hits/minute) steel has a microstructure that is highly fibered. The Manganese Sulfide (MnS) stringers are surrounded by a soft ductile ferrite phase. Photograph 1 at 100X, illustrates the woody, fibrous fracture characteristic of a transverse crack. Photograph 2 shows the inside diameter where the crack initiated at 760X magnification. At magnifications above $2000 \mathrm{X}$ it can be clearly seen, Photograph 3, that ductile micro-void coalescence, (MVC), has preceded the macroscopic fracture. In fact areas of serpentine glide are observed around the interfacial fractures of the ferrite encapsulated inclusions.

An example of adiabatic shear in Cartridge Brass is shown in Photograph 4. During the discussion of the steel failure which has been incorrectly diagnosed as an impact fatigue failure, a similar example of adiabatic shear will be shown to illustrate the value of a complete microstructural analysis of a failure. It is essential to conduct a complete analysis of the subject microstructure in order to arrive at the proper determination of failure mode. Photograph 4 is taken from a $5.56 \mathrm{~mm}$ center fire cartridge Lake City Army Ammunition Plant, SCAMP.

\section{References}

[1] B. L. Gabriel, ASM, SEM: A user's Manual for Materials Science, Metals Park, (1985) 100 [2] R. W. Hertzberg, K.S.Vecchio, and F.E. Schmidt, Jr., ASTM J O T E, Philadelphia, PA September (1989) 261.

[3] K.S. Vecchio, R.W. Hertzberg, and F.E. Schmidt, Jr., ASTM J O T E, Philadelphia, PA September (1989) 267. 


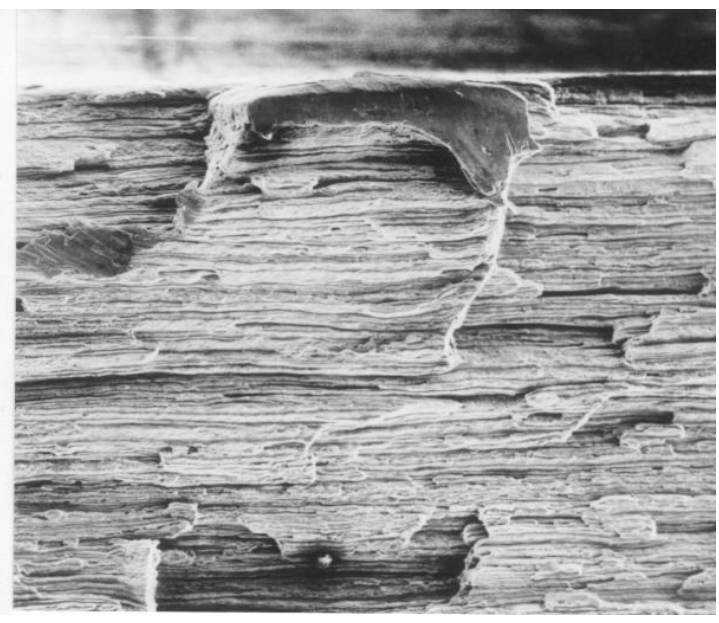

Photograph 1

Fibrous Characteristic of Transverse Crack (Magnification: 100X)

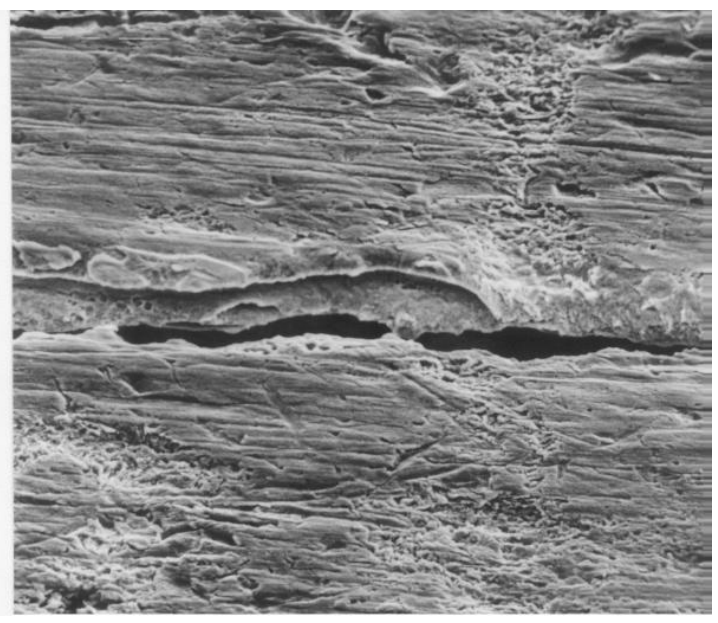

Photograph 2

Crack Initiation on Inside Diameter (Magnification: 760X)

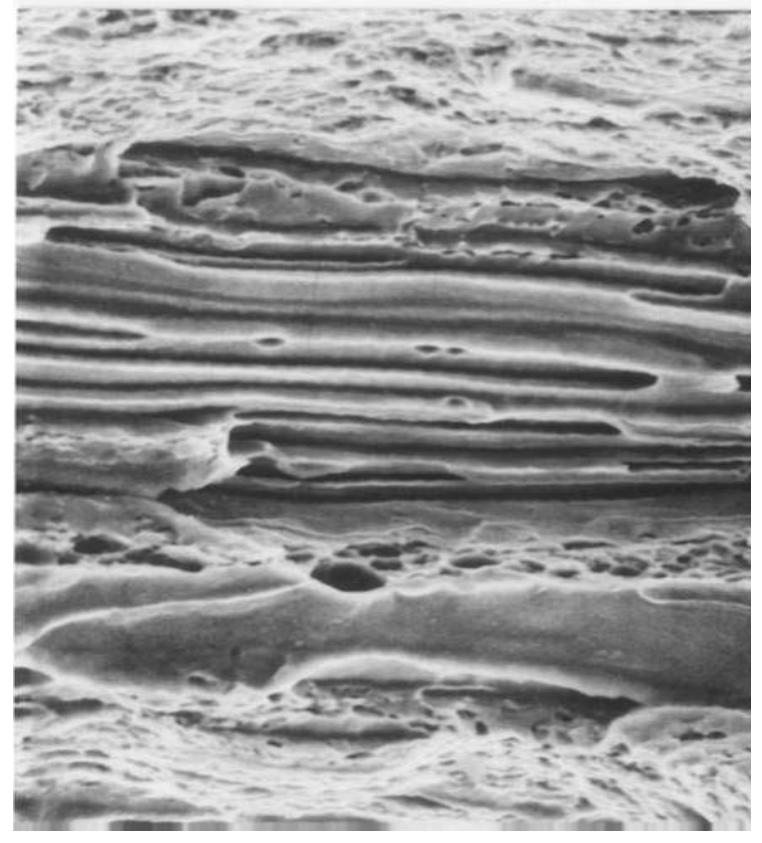

Photograph 3

Ductile Micro-voids (MVC) and Serpentine Glide around the $\mathrm{MnS}$ inclusions (Magnification: 2000X)

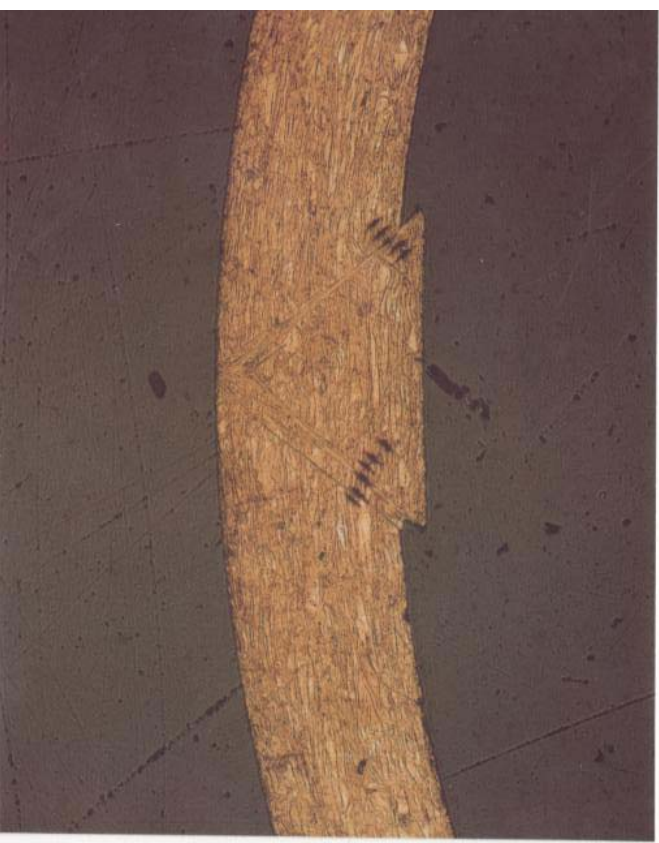

Photograph 4 Adiabatic Shear in $5.56 \mathrm{~mm}$ Cartridge Brass 70:30 Center Fire Case (Magnification: 75X) 